\title{
Comparación de la resistencia a la alteración por niebla salina de dos calizas utilizadas en la construcción mediante métodos ponderales, métodos acústicos y tratamiento de imágenes
}

\author{
Comparaison de l'altérabilité au brouillard salin de deux pierres \\ calcaires de construction au moyen de mesures pondérales \\ acoustiques et par traitement d'images
}

BIRGINIE, J.M.*; RIVAS, T.**; PRIETO, B. ${ }^{\star *}$; AUGER, F. *

${ }^{\star}{ }^{\star}$ Laboratoire de Construction Civile et Maritime (IUT)

FRANCIA

Fecha de recepción: 14-09-99

$\left.{ }^{(\star \star}\right)$ Dpto. de Edafología. Fac. de Farmacia. Univ. de Santiago

Fecha de aceptación: 21-03-00

ESPAÑA

\section{RESUMEN}

Se presentan los resultados de un estudio comparativo de la resistencia a la alteración en atmósfera de niebla salina de dos rocas calizas utilizadas en la construcción de monumentos en Francia y en España. Las modificaciones experimentadas por las probetas durante este ensayo se han valorado utilizando tres métodos: la medida de la evolución del peso, la medida de la velocidad de propagación de ultrasonidos y el análisis visual de las modificaciones superficiales por un sistema de barrido mediante luz láser de las superficies (método de triangulación luz láser-cámara) y posterior tratamiento de imágenes. La comparación de los resultados obtenidos muestra que los tres métodos ofrecen una información complementaria que permite caracterizar de manera precisa el proceso de alteración y su evolución durante el ensayo. Es, sin embargo, el método de análisis mediante el sistema láser-cámara el que ofrece más posibilidades para describir y comparar de manera objetiva la morfología y la evolución de la desagregación arenosa observada sobre las dos rocas calizas alteradas artificialmente.

\section{RESUME}

Nous présentons les résultats d'une étude comparative de la résistance à l'altération au brouillard salin de deux pierres utilisées dans la construction de monuments en France et en Espagne. Les transformations subies par les échantillons au cours de l'essai de vieillissement ont été évaluées en utilisant trois méthodes: l'évolution de la masse, la mesure de la vitesse de propagation d'ultrasons et l'analyse visuelle automatisée des désagrégations superficielles au moyen d'un système de balayage par plan laser de la surface (triangulation laser-caméra). La comparaison de l'ensemble de ces résultats démontre que les trois méthodes non destructives fournissent des informations complémentaires qui permettent de caractériser les processus d'altération et leur évolution. C'est néanmoins l'analyse visuelle des surfaces par le système laser-caméra qui offre le plus de possibilités pour décrire et comparer. tant sur le plan quantitatif que qualitatif, l'évolution des désagrégations sableuses observées sur les deux pierres calcaires vieillies artificiellement par brouillard salin.

\section{INTRODUCCIÓN}

Uno de los mecanismos de deterioro de efectos mas graves que actúan en los materiales rocosos es la cristalización de sales solubles dentro del espacio poroso $(1,5,11)$; las sales solubles en las rocas utilizadas en los monumentos tiene varios orígenes;

\section{INTRODUCTION}

L'un des mécanismes de détérioration qui affecte le plus les matériaux pierreux utilisés dans la construction résulte de la cristallisation de sels solubles à l'intérieur de l'espace poreux $(1,5,11)$. La présence des sels dans les pierres des monuments 
entre ellos cabe destacar los aportes procedentes del mar, ya sea por vía húmeda (niebla salina) o seca. Los efectos de la acción de las sales de origen marino en los monumentos se manifiestan sobre todo por la desagregación arenosa de la superficie de la roca, de efectos en muchos casos tan devastadores que hacen peligrar el valor artístico de la obra $(5,9,12,13)$.

Los ensayos de alteración artificial en laboratorio, en los cuales se introducen los factores de deterioro, en este caso, las sales, y las condiciones ambientales a las que está sometido el edificio constituyen una metodología básica encaminada a reproducir en condiciones controladas el deterioro observado en los monumentos y a establecer el mecanismo de alteración $(1,3,5,12)$.

Con el propósito de evaluar el grado de deterioro de los materiales rocosos durante estos ensayos, pueden ser aplicados varios métodos de medida, por ejemplo, la variación de masa, la medida de propiedades mecánicas o la velocidad de propagación de ultrasonidos (2). Sin embargo, estas mediciones, debido a que afectan de manera global a las probetas, no permiten analizar específicamente los fenómenos de deterioro, ya que éstos tienen lugar sobre todo en las zonas más cercanas a la superficie.

En este trabajo, la alteración provocada por la acción de la niebla salina en dos tipos de piedra caliza utilizadas en la construcción de monumentos es evaluada a través de tres métodos no destructivos. Los dos primeros, la medida de la variación de peso y de la velocidad de propagación de ultrasonidos, caracterizan el estado global del volumen de la probeta $(2,4)$; la velocidad de propagación, además, ofrece información acerca del grado de cohesión de la roca. El tercer método consiste en un barrido de la superficie alterada de las probetas mediante un sistema provisto de una cámara y de un haz de luz láser (7) que permite el análisis de la evolución del deterioro superficial. El objetivo de esta experimentación es el de comparar la información que dan los tres métodos propuestos y establecer su utilidad para permitir una mejor comprensión del fenómeno de deterioro que se produce en los materiales.

Las rocas seleccionadas, Richemont, utilizada en la restauración de monumentos antiguos en Francia, y una variedad de la denominada Piedra del País, material de construcción de la Catedral de León (España) (11) se han sometido a un ensayo de simulación de alteración por niebla salina el cual, en trabajos anteriores $(1,3)$, ha demostrado ser un sistema que reproduce de manera muy fiel el proceso de deterioro que sufren los materiales rocosos sometidos a la acción de aerosoles marinos. a plusieurs origines parmi lesquelles les apports marins, que ce soit sous forme de solution (brouillard salin) ou sous forme d'aérosols. L'action de ces sels se manifeste surtout par des désagrégations sableuses et alvéolaires qui endommagent profondément la surface de la pierre au point de faire disparaître les parements artistiques des édifices (5, 9, 12, 13).

Les essais d'altération artificielle réalisés en laboratoire visent à recréer dans un espace confiné, les facteurs de détérioration, dans notre cas les sels, et les conditions d'environnement auxquels sont habituellement exposés les édifices $(1,3,5,12)$. Cette méthodologie de base permet de reproduire et d'accélérer en ambiance contrôlée les dégradations observées sur site et d'étudier les mécanismes d'altération.

Afin d'évaluer l'état de détérioration des matériaux durant ces essais, divers moyens de mesure non destructifs peuvent être utilisés, dont par exemple : la variation de masse, la détermination de propriétés mécaniques ou la mesure de la vitesse de propagation d'ondes ultrasonores (2). Cependant, étant donné qu'il $s$ 'agit là de propriétés déterminées de manière globale sur l'ensemble de l'échantillon, la plupart de ces méthodes ne donnent pas en général la possibilité d'analyser spécifiquement les phénomènes de détérioration qui se développent surtout dans les zones voisines de la surface.

Dans le cadre de ce travail, l'altération liée aux effets du brouillard salin sur deux types de pierres calcaires utilisées dans la construction, a été évaluée à travers trois méthodes d'analyse non destructives. Les deux premières, la mesure de la variation de la masse et la mesure de la vitesse de propagation du son, caractérisent globalement l'échantillon par une donnée physique d'ensemble (2, 4); la vitesse du son, aussi, donne information sur la cohésion interne des roches. La troisième méthode consiste à balayer la surface altérée à l'aide d'un système lasercaméra afin de permettre une analyse détaillée de l'évolution de la dégradation (7). L'objectif de l'expérimentation présentée est de comparer les informations données par les trois méthodes d'analyse proposées en montrant que leur complémentarité permet de mieux comprendre les phénomènes de détérioration qui se produisent dans les matériaux.

Les deux pierres sélectionnées, le Richemont, utilisé notamment pour la restauration de monuments en France et une variété ocre de la Pierre de Pays, matériau de construction de base de la Cathédrale de León (Espagne) (11), ont été soumises à un même essai de simulation d'altération par brouillard salin dont des études préalables $(1,3)$ ont montré qu'il reproduisait de manière très fidèle le processus de détérioration dont souffrent les matériaux pierreux sur site sous l'effet des aérosols marins. 


\section{MATERIAL Y MÉTODOS}

Las dos rocas seleccionadas se describen de la manera siguiente:

. La roca denominada Richemont es una caliza oolítica de grano fino, equigranular, de color blanco grisáceo que pertenece al Cretácico Superior Turoniense. Posee un valor de porosidad accesible del $25 \%$. Procede de la localidad francesa de Avy (Departamento de La Charente Maritime, Francia) y constituye un material muy utilizado en la restauración de los monumentos románicos de la región.

. La variedad rojiza del material rocoso denominado localmente Piedra del País procedente de la provincia de León $(10,11)$; se trata de una caliza heterogénea de grano fino, de coloración amarillo-rojiza debido a la presencia abundante de oxihidróxidos de hierro.

Petrográficamente está compuesta por calcita esparítica embebida en una matriz detrítica compuesta por arcilla y cuarzo de tamaño de grano variable. La calcita esparítica aparece a veces formando cavidades de tamaño variable entre $0,05-5 \mathrm{~mm}$. Su porosidad accesible al agua corresponde a un $12 \%$.

De cada roca se han preparado cinco probetas cilíndricas de $50 \mathrm{~mm}$ de diámetro y $100 \mathrm{~mm}$ de altura procedentes del mismo bloque, cortadas perpendicularmente al plano de estratificación de cantera.

\section{Ensayo de alteración artificial}

Las probetas se sometieron a un ensayo de alteración por niebla salina durante 36 días, utilizando un simulador controlado electrónicamente descrito en $(1,3)$ el cual realiza ciclos de 30 minutos de duración de los cuales 1 minuto corresponde a una pulverización con niebla salina y 29 minutos un secado a ventilación forzada a $40 \pm 1{ }^{\circ} \mathrm{C}$. La niebla salina se produce haciendo pasar una corriente de aire a flujo constante a través de un pulverizador normalizado que aspira, por efecto venturi, agua de mar. Las probetas se sitúan sobre un soporte giratorio que, durante el episodio de pulverización de niebla, realiza cuatro giros completos, para asegurar una repartición homogénea de la niebla salina sobre la superficie total de las probetas. De esta manera, las probetas reciben una cantidad de disolución similar del orden de 0,4 gramos repartida por toda la superficie lateral. Una vez al día, la niebla salina es reemplazada por un lavado automático de la superficie de las probetas con agua destilada, con el propósito de eliminar las posibles deposiciones salinas que se produzcan en la superficie.

\section{MATÉRIAUX ET MÉTHODES}

Les deux pierres calcaires étudiées se décrivent de la manière suivante :

- La pierre de Richemont est un calcaire oolithique à grains fins, iso-granulaire, de teinte gris blanc qui s'est formé au Crétacé Supérieur Turonien. Sa porosité accessible à l'eau est de $25 \%$. Elle provient de la localité française d'Avy dans le Département de Charente Maritime (France) et constitue l'un des matériaux d' uvre utilisés dans la restauration de nombreux monuments.

\section{. La variété ocre de la pierre de la région de León} (Espagne), dénommée localement Pierre du Pays $(10,11)$, est un calcaire hétérogène, à grains fins, de couleur jaunerouge due à la présence d'oxyde de fer, comportant des zones de recristallisation de calcite. Sur le plan pétrographique, elle présente des zones sparitiques noyés dans un ensemble détritique, micritique de calcite, quartz, argile de tailles et de formes variables. Localement, apparaissent des cavités de dimension comprise entre 0,05 et $5 \mathrm{~mm}$ remplies de calcite de recristallisation. La porosité accessible de cette pierre correspond $\dot{a} 12 \%$.

Pour chaque type de roche, cinq éprouvettes cylindriques de $50 \mathrm{~mm}$ de diametre et $100 \mathrm{~mm}$ de haut, provenant respectivement du même bloc ont été carottées dans la direction perpendiculaire au plan des strates.

\section{Essai de simulation}

Les éprouvettes sont soumises durant 36 jours à un essai d'altération par brouillard salin dans l'un des simulateurs décrits dans les références $(1,3)$. Ce type de simulateur, contrôlé par un système automatisé, réalise des cycles d'une durée de 30 minutes comprenant une phase d'une minute de pulvérisation de brouillard suivi d'un séchage par ventilation régulé à $40 \pm 1^{\circ} \mathrm{C}$. Le brouillard salin est produit par aspiration d'eau de mer dans un pulvérisateur normalisé alimenté par un flux d'air sous pression (effet venturi). Une fois par jour, la phase de brouillard salin est remplacée par un lavage à l'éu distillée de l'éprouvette, cela dans le but d'éliminer les dépôts de sel qui durcissent à la surface. Les éprouvettes sont placées sur un support en rotation à l'intérieur du simulateur de façon à assurer une répartition homogène de la solution sur les faces latérales des pierres durant la phase de pulvérisation de brouillard salin. Les échantillons. reçoivent ainsi sur leur surface une quantité de solution de l'ordre de $0,4 \mathrm{~g}$ à chaque pulvérisation. 
Regularmente, las probetas son extraídas del sistema y sobre ellas se aplican los tres siguientes métodos de control no destructivos:

\section{Métodos de control}

Los métodos aplicados para analizar el deterioro provocado por el ensayo son:

. Medida de la variación de peso de las probetas

. Medida de la velocidad de propagación de ondas acústicas longitudinales (ondas $\mathrm{Vp}$ de compresión) por transparencia mediante un equipo detector PUNDIT. La frecuencia de emisión y de recepción acústica corresponde a $54 \mathrm{KHz}$, transmitiéndose las ondas paralelamente al eje longitudinal de las probetas las cuales se colocan en un soporte fijo a presión constante.

\section{- Análisis superficial del deterioro por tratamiento de} imágenes

Los dos primeros análisis se realizan de manera regular cada día, y, dada la elevada sensibilidad de estos métodos ante la presencia de agua en los materiales, se determinan respetando un tiempo mínimo de secado de 25 minutos tras la última pulverización diaria.

El análisis de las superficies por tratamiento de imágenes se efectúa cada cuatro días utilizando un sistema controlado electrónicamente y manejado a través de un ordenador (7). El sistema esta compuesto por una cámara oscura en donde se fija la probeta cilíndrica sobre un soporte y se hace girar sobre su eje central ante un
Les éprouvettes sont retirées régulièrement $d u$ simulateur pour réaliser les trois contrôles non destructifs proposés:

\section{Méthodes de contrôle}

Les moyens mis en uvre pour contrôler l'état de la détérioration sont les suivants :

. La mesure de la variation de masse des éprouvettes,

. La mesure de la vitesse de propagation des ondes ultrasonores longitudinales (ondes $V p$ de compression) par transparence à l'aide d'un équipement de détection PUNDIT, l'émission et la réception acoustique, de fréquence de $54 \mathrm{KHz}$, s'effectuant suivant l'axe longitudinal de l'éprouvette fixée sur un banc à pression constante $(2,4)$.

\section{. L'analyse superficielle de la détérioration par} traitement d'images et balayage par faisceau laser.

Ces deux premières mesures sont réalisées de manière journalière en retirant les échantillons strictement dans les 5 dernières minutes de séchage en raison de la sensibilité de ces mesures à la présence d'eau dans la pierre.

La dernière analyse s'effectue tous les quatre jours de simulation environ au moyen d'un système laser-caméra piloté par ordinateur (7). Le système se compose d'une chambre obscure à l'intérieur de laquelle l'éprouvette est fixée sur un support circulaire devant un système optique associant une caméra CCD et un plan de lumière

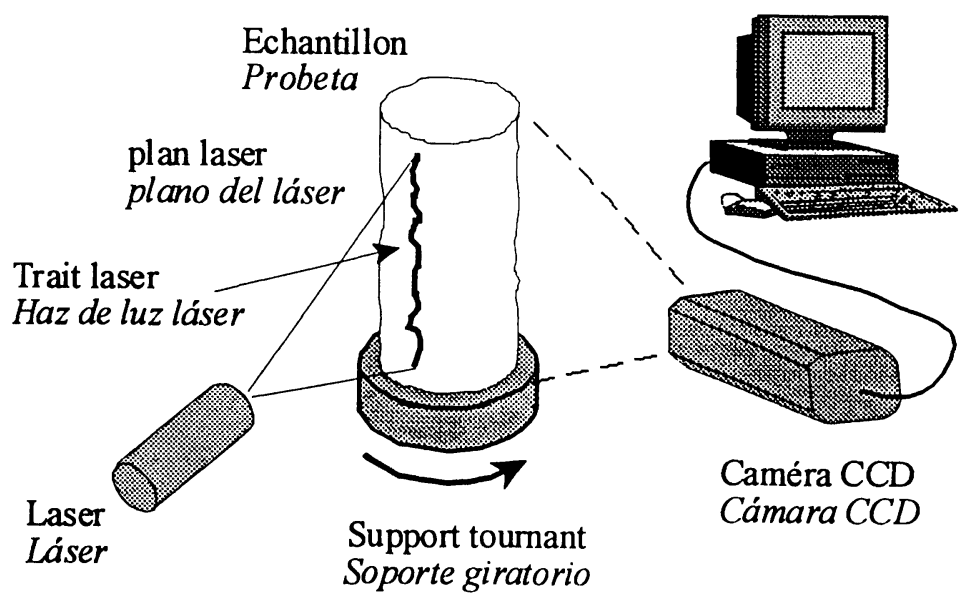

Figura 1.- Esquema del dispositivo de análisis visual de una probeta cilíndrica.

Figure 1.- Schéma du dispositif d'analyses visuelles d'un échantillon cylindrique. 
sistema óptico compuesto por una cámara CCD y un proyector de haz de luz láser (Figura 1). La información obtenida por la cámara (el trazo de la luz láser sobre la superficie de la probeta) es enviada a un ordenador y procesada mediante un programa diseñado específicamente para este análisis. El sistema ofrece dos tipos de información (7):

- Información relativa al relieve de la superficie de la probeta. La extracción de la información del relieve se basa en un método de triangulación entre el plano de emisión del haz de luz láser y el plano de la proyección del mismo sobre la superficie de la probeta vista a través de la cámara. De esta forma, conociendo la orientación de los ejes ópticos del haz de láser y de la cámara, se deduce mediante una relación geométrica la posición en tres dimensiones de cada punto de la superficie de la probeta $(6,7)$.

. Información relativa a las propiedades de reflexión de la luz láser por la superficie de la probeta. La intensidad luminosa del trazo láser sobre cada punto de la superficie de la probeta se transforma en un valor numérico que varía de 0 a 255 .

Ambas informaciones son extraídas y registradas en forma de matrices de datos numéricos de un tamaño de $200 \times 134$ que permite respetar la relación dimensional entre la altura $(100 \mathrm{~mm})$ y la circunferencia ( $50 \mathrm{~mm} \times \Pi \equiv 150 \mathrm{~mm}$ ) de la probeta. El estudio de estas matrices se aborda desde dos aspectos:

1. Análisis cualitativo del deterioro: para ello, las matrices de datos del relieve son presentadas de dos maneras diferentes:

- mapas topográficos del relieve en los que se representan sobre un gráfico en tres dimensiones los valores brutos de las matrices sin transformar,

- mapas de niveles de gris en los que se asocia un nivel de gris a un nivel de relieve. Esta representación se realiza sustrayendo a cada matriz numérica obtenida cada cuatro días del ensayo la matriz numérica inicial correspondiente al relieve de la probeta sin alterar; de esta forma se pone en evidencia únicamente la alteración desarrollada durante el ensayo.

2. Análisis cuantitativo del deterioro. Para ello, se ha determinado la desviación típica del conjunto de valores numéricos de cada matriz de reflexión y de relieve. El análisis de este parámetro a lo largo del ensayo informa acerca del grado de alteración sufrida en el tiempo. En el caso de las matrices de relieve nos da una idea de la evolución de la rugosidad y, en el laser (Figure 1). Le balayage de la surface est assuré en faisant tourner le support à l'aide d'un moteur pas à pas. L'information obtenue sous forme d'images digitalisées est transmise à un ordinateur qui repère, à l'aide d'un programme spécifique, le trait laser apparaissant sur l'image. Le système fournit alors deux types d'information (7) :

\section{. L'information relative au relief de la surface de} l'échantillon. L'extraction du relief est basée sur un principe de triangulation entre le point d'émission de la source laser, le plan de lumière et le point de projection du faisceau sur la surface de l'éprouvette vue à travers la caméra. De cette manière, connaissant l'orientation de chaque système optique, on déduit par une relation géométrique la position en trois dimensions des points de la surface $(6,7)$.

- L'information relative à l'évolution des propriétés de réflexion de la lumière laser de la surface. L'intensité lumineuse maximale du trait laser est restituée sous la forme d'une valeur numérique variant de 0 à 255.

L'ensemble de l'information visuelle est enregistré sous la forme de matrices de données numériques de taille $200 \times 134$ correspondant au rapport dimensionnel de la circonférence ( $\Pi \times 50 \mathrm{~mm} \cong 150 \mathrm{~mm}$ ) sur la hauteur (100 mm) des échantillons. L'exploitation de ces matrices a été réalisée selon les deux approches suivantes :

\section{L'évaluation qualitative de l'altération en utilisant} deux types différents de représentation du relief de la surface

- des cartes topographiques qui montrent sur un graphique $3 D$ les données brutes de la matrice.

- des images en niveaux de gris où les niveaux du relief sont associés à une échelle de couleur. Dans cette seconde représentation, on soustrait à chaque matrice de relief la matrice initiale correspondant $\dot{a}$ l'échantillon non altéré de façon à mettre en évidence uniquement l'altération survenant au cours de l'essai.

2. L'évaluation quantitative de l'altération en calculant l'écart type des données numériques sur chaque matrice (relief et réflexion de lumière laser). L'analyse de ce paramètre au cours de la simulation rend compte de l'évolution du niveau d'altération subi par la surface des échantillons. Pour le relief, le paramètre statistique utilisé peut être assimilé à un critère de rugosité de surface. Pour la réflexion, le paramètre 
caso de la reflexión, de la evolución de las propiedades ópticas de reflexión de la superficie de las probetas.

Finalmente se ha determinado el contenido de ion cloruro y sodio en extractos acuosos de muestras recogidas a distintas profundidades desde la superficie expuesta de las probetas. Para ello, el polvo, seco hasta peso constante, resultado de la perforación realizada cada $3 \mathrm{~mm}$ de profundidad se agita durante 15 minutos en $100 \mathrm{ml}$ de agua destilada. En la suspensión se determina el contenido en cloruro mediante valoración con $\mathrm{AgNO}_{3}$ (Método de Mohr) utilizando cromato potásico como indicador.

\section{RESULTADOS}

\section{Evolución del peso}

La Figura 2, que representa la variación del peso de las probetas de Richemont (2-b) y de León (2-a) a lo largo statistique donne des informations sur les modifications des propriétés de réflexion des surfaces.

A la fin des essais de vieillissement, des mesures de concentration de $\mathrm{NaCl}$ ont été réalisées sur les échantillons en effectuant des prélèvements à différentes profondeurs. Après broyage, ces prélèvements sont introduits dans un Becher contenant $100 \mathrm{ml}$ d'eau distillée, puis mélangés à température ambiante, avec un agitateur magnétique durant 15 minutes pour dissoudre les sels solubles. Les concentrations sont obtenues par la Méthode de Mohr (titrage au nitrate d'argent en présence de chromate de potassium).

\section{RÉSULTATS}

\section{Evolution de la masse}

La Figure 2 qui présente graphiquement la variation de la masse (en \%) des cinq éprouvettes de
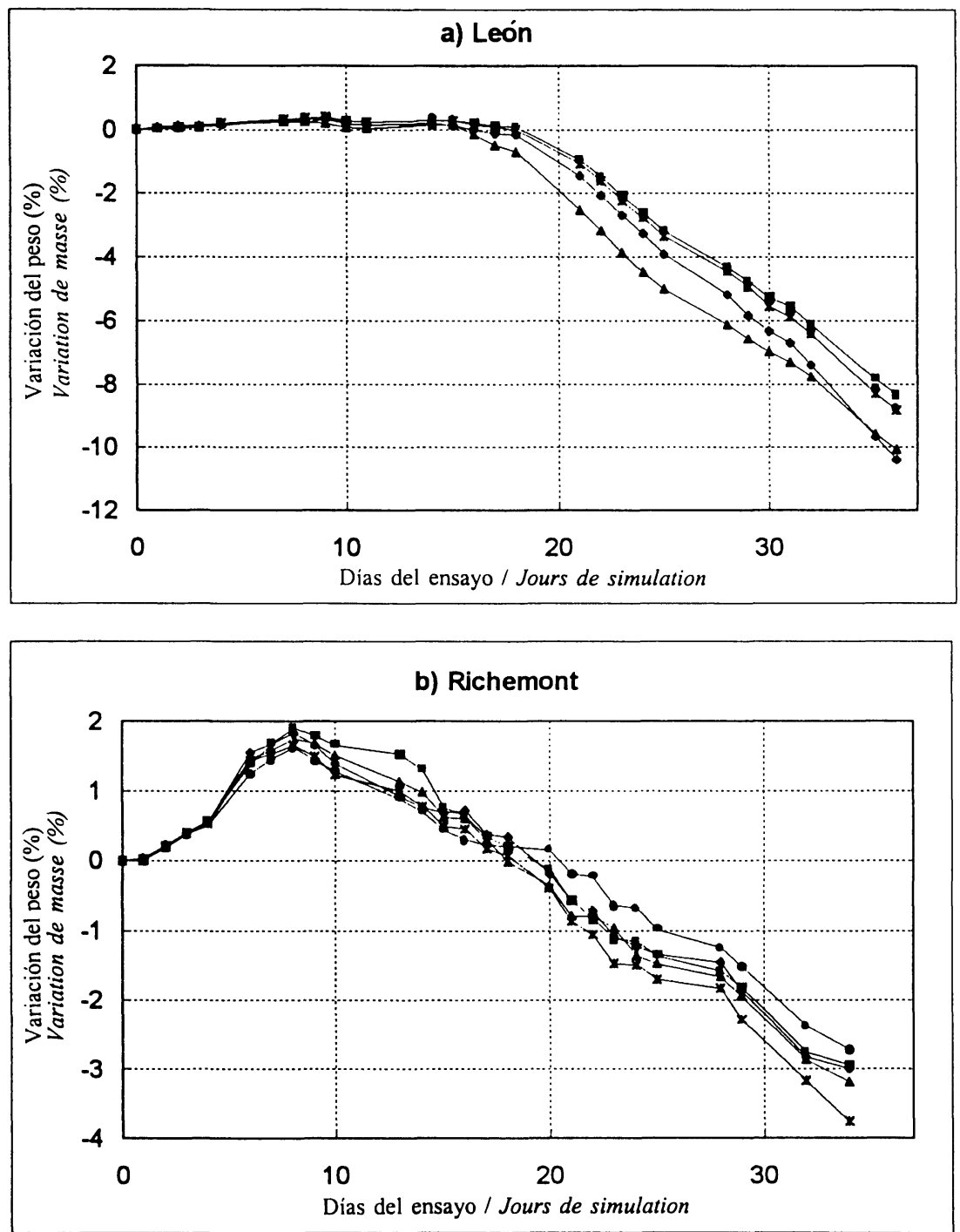

Figura 2.- Variación del peso de las probetas de León (a) y de Richemont (b) durante el ensayo de alteración por niebla salina.

Figure 2.- Courbes d'évolution de masse des échantillons de León (a) et de Richemont (b) au cours de la simulation 
de la simulación, pone en evidencia un comportamiento bastante similar entre las probetas de la misma roca y un comportamiento diferente entre ambas rocas.

El peso de las probetas de León no muestra ninguna variación hasta 15 días después de comenzado el ensayo, momento a partir del cual el peso decrece de manera continua en un $0,5 \%$ al día. Las primeras manifestaciones de deterioro surgieron en forma de desagregación arenosa varios días antes del comienzo de la pérdida de peso, afectando a la superficie de la roca de manera muy localizada. El deterioro fue acentuándose progresivamente en forma de alveolos más o menos profundos. Al final del ensayo, la pérdida de peso sufrida por estas probetas correspondió a un 10\% del peso inicial. Es necesario destacar la ausencia durante todo el ensayo de incremento de peso.

Las probetas de la piedra de Richemont muestran, por el contrario, una ganancia de peso de más del 1,5\% durante la primera semana del ensayo, que refleja una acumulación significativa de disolución. A partir del décimo día del ensayo, el peso comenzó a disminuir coincidiendo con el desencadenamiento de desagregación arenosa. La pérdida de peso continúa con una tasa diaria del $0,15 \%$, tres veces menor que la correspondiente a la roca de León.

Es de destacar que el color de esta roca se alteró bruscamente hacia un tono más oscuro tras cinco días de comenzado el ensayo, lo que se atribuyó a la oxidación de los minerales de hierro que posee esta roca.

\section{Evolución de la velocidad de propagación de ultrasonidos}

Con respecto a esta segunda caracterización, el comportamiento de los dos tipos de piedra difiere notablemente (Figura 3-a). En el caso de la roca de León, la velocidad de ultrasonidos muestra una débil variación con una ligera tendencia a disminuir tras tres semanas de ensayo. En Richemont (Figura 3-b), la velocidad de propagación de ultrasonidos se mantiene constante alrededor de $3.100 \mathrm{~m} / \mathrm{s}$ durante los cuatro primeros días del ensayo; después sufre un descenso significativo en las cinco probetas que concuerda con el momento en que la acumulación de disolución en éstas se hace importante, superior al $1 \%$ con respecto al peso inicial (Figura 2-b). La velocidad de propagación se estabiliza posteriormente alrededor de $2.750 \mathrm{~m} / \mathrm{s}$ sin que la pérdida de masa que se produce en esta fase del ensayo afecte a la evolución de esta propiedad.
Richemont (2-b) et de León (2-a) durant la simulation, met en évidence l'évolution très similaire des échantillons de même nature mais aussi une différence de comportement très marquée entre les deux types de pierre.

La masse des éprouvettes de León n'évolue pratiquement pas jusqu'au $15^{\text {ème }}$ jour de la simulation, puis décroît de manière continue avec un taux d'évolution d'environ 0,5\% par jour. Les premiers signes de désagrégation de la surface de la pierre sont apparus d'abord de manière très localisée et ont précédé de quelques jours la diminution de la masse. La détérioration s'est ensuite accentuée sous la forme d'une désagrégation alvéolaire et, au terme de l'essai, la perte de masse des échantillons a atteint prés de $10 \%$ de la masse initiale. Soulignons la quasi-absence de gain de poids tout au long de l'essai, dans le cas de la pierre de León.

Les échantillons de Richemont ont en revanche montré durant la première semaine de l'essai un gain de poids de plus de 1,5\% qui correspond à l'accumulation d'une quantité significative de solution. A partir du $10^{\text {ème }}$ jour de la simulation, une forte diminution de la masse s'est produite au moment où les désagrégations ont commencé à affecter les surfaces des échantillons. La perte de poids s'est ensuite établie à une moyenne d'environ 0,15\% par jour, soit près de trois fois moins que celle constatée sur les pierres de León.

Notons que la teinte initialement blanche des échantillons de Richemont s'est brusquement assombrie dès la première semaine de l'essai de vieillissement. Cette variation de teinte est due à l'oxydation de minéraux ferreux disséminés dans la pierre.

\section{Evolution de la vitesse du son}

Par rapport à cette seconde caractérisation, le comportement des deux types de pierre diffère aussi de manière importante (Figure 3-a). Dans le cas de la pierre de León, les courbes de mesure de la vitesse $d u$ son montrent une faible évolution avec une tendance à diminuer après trois semaines d'essai. En ce qui concerne les échantillons de Richemont (Figure 3-b), la vitesse du son s'est maintenue à $3.100 \mathrm{~m} / \mathrm{s}$ durant les 4 premiers jours d'essai, puis elle a très vite chuté au moment où l'accumulation de masse est devenue importante, supérieure à $1 \%$ du poids initial des éprouvettes (Figure 2-b). La vitesse $d u$ son s'est ensuite stabilisée à environ $2.750 \mathrm{~m} / \mathrm{s}$ sans que la perte de masse ne vienne affecter cette seconde étape d'évolution. 

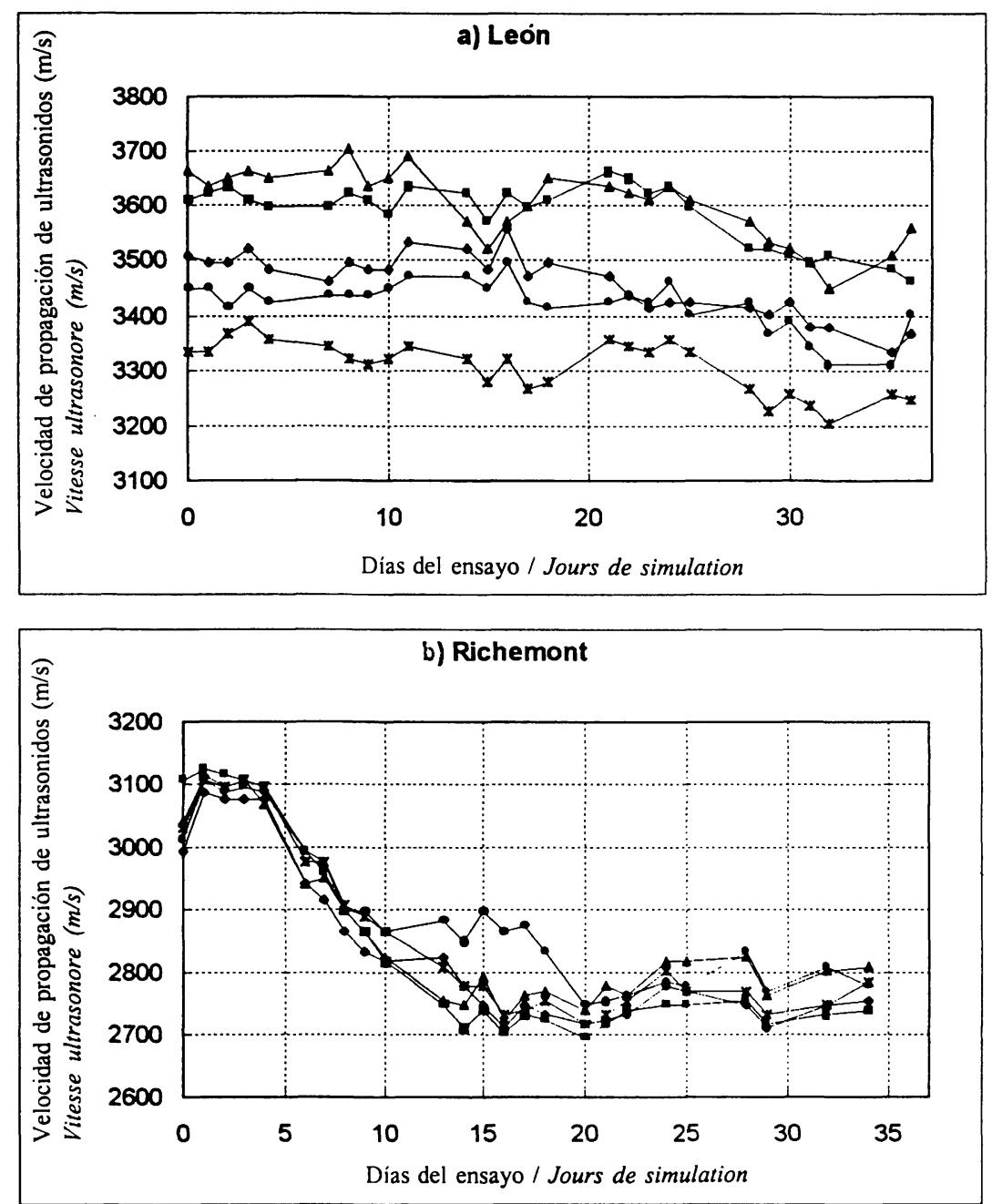

Figura 3.- Evolución de la velocidad de propagación de ultrasonidos en las probetas de L_cón (a) y de Richemont (b) sometidas al ensayo de alteración por niebla salina.

Figure 3.- Courbes d'évolution de masse de la vitesse du son des échantillons de León (a) et de Richemont (b) au cours de l'essai d'altération par brouillard salin.

Es destacable la fuerte dispersión de los valores de propagación acústica entre las probetas de la piedra de León, que se atribuye a la importante heterogeneidad textural de la piedra de León, caracterizada por la existencia de cavidades de calcita recristalizada repartidas de forma aleatoria.

Finalizado el ensayo, las probetas de ambas rocas se secaron en estufa a $40^{\circ} \mathrm{C}$ hasta peso constante con el propósito de evaluar, tras un secado completo, la influencia de la cristalización de la sal presente en la roca en la velocidad de propagación de ultrasonidos. En la Tabla 1 se presentan los valores medios de la velocidad de propagación de cada roca medidos antes del ensayo, al final del mismo y tras el secado en estufa hasta peso constante.

La evolución de la velocidad de propagación de ultrasonidos observada en ambas rocas durante el
La forte dispersion des résultats de la mesure acoustique pour la pierre de León face à ceux du Richemont peut être attribuée à l'hétérogénéité de la texture de la pierre de León qui comprend des inclusions de calcite cristallisée réparties aléatoirement dans le matériau.

A la fin de l'essai, les échantillons ont été séchés en étuve à $40{ }^{\circ} \mathrm{C}$ jusqu'à stabilisation de leur poids afin d'évaluer l'influence de la cristallisation des sels sur les propriétés acoustiques des matériaux. Ainsi, le tableau 1 compare les valeurs moyennes de la vitesse du son des échantillons mesurées au début de l'essai, au dernier jour de l'essai et après le séchage en étuve.

La tendance d'évolution de la vitesse du son qui a été observée sur les deux pierres durant l'essai de 
Velocidad de propagación de ultrasonidos de León y Richemont antes y después del ensayo y tras secado hasta peso constante en estufa. valores medios de las cinco probetas de cada roca

(Valeurs moyennes pour les cinq éprouvettes de León et Richemont de la vitesse mesurées au début et au terme de l'essai de vieillissement et après un séchage en étuve)

\begin{tabular}{|c|c|c|}
\hline Velocidad de propagación (m/s) (Vitese du son $(\mathrm{m} / \mathrm{s})$ ) & León & Richemont \\
\hline Inicial(Initiale) & 3508 & 3086 \\
Finalizado el ensayo (Finale après l'essai) & 3289 & 2639 \\
Secado en estufa (Finale après l'essai) & 3546 & 3311 \\
\hline
\end{tabular}

ensayo corresponde a la constatada generalmente en rocas de naturaleza caliza durante un proceso de absorción libre de agua $(4,8)$. Durante la absorción de agua desde el estado seco hasta la completa saturación, la velocidad de propagación de ultrasonidos sufre una disminución más o menos acentuada dependiendo de la porosidad de la roca, y cuya cinética está influenciada por la configuración del espacio poroso. En este trabajo, las medidas de ultrasonidos realizadas durante el ensayo conciernen al volumen total de las probetas. Por tanto, únicamente cuando la disolución salina penetra hasta profundidad en la roca, alcanzando el interior de las probetas, se detectan variaciones en los valores de propagación.

Aunque en este trabajo no se ha realizado una caracterización del sistema poroso de las rocas, para interpretar el comportamiento de las mismas durante este ensayo es suficiente, a nuestro juicio, la información que ofrecen la medida de la variación de peso, la medida de la variación de ultrasonidos y los valores de la porosidad accesible.

La roca de León no experimenta un incremento de peso tras los primeros episodios de pulverización de niebla y tampoco un descenso en los valores de velocidad de propagación lo que indica que la disolución no alcanzó el interior de la probeta. Ambos hechos muestran que la absorción de la disolución por esta roca es débil y superficial. Richemont, más porosa que León, muestra, por el contrario, un rápido incremento de peso desde el inicio del ensayo, coincidente con un fuerte descenso en la velocidad de ultrasonidos la cual, al final del ensayo, se estabiliza en un valor un 10-15\% más bajo que el valor inicial antes del ensayo. Por tanto, Richemont absorbe más cantidad de disolución y ésta llega a impregnar el espacio poroso del interior de la probeta.

Durante el ensayo, pues, la cinética de evaporación y de humectación de León afecta a una zona muy próxima a vieillissement correspond à celle généralement constatée sur les pierres calcaires lors d'une phase d'absorption d'eau $(4,8)$. Lorsque la pierre passe de l'état sec à l'état saturé, la vitesse du son diminue de manière plus ou moins importante en fonction de la porosité accessible du matériau et avec une rapidité qui dépend de la conformation du système poreux des pierres (taille et connectivité des pores). Par ailleurs, la mesure acoustique que nous pratiquons concerne l'échantillon dans sa globalité et ne peut varier que si la pierre a été mouillée à coeur.

Bien que dans ce travail, nous n'ayons pas réalisé d'étude détaillée sur la configuration porosimétrique de ces pierres pour expliquer les processus de diffusion et d'altération, les données concernant les variations de la vitesse du son et de la masse, et la valeur de la porosité accessible à l'eau sont néanmoins suffisantes pour permettre l'interprétation de ces phénomènes dans leur globalité.

Dans le cas par exemple de la pierre de León, l'augmentation de la masse au début de l'essai a été faible et la vitesse du son n'a que peu évolué durant l'essai. L'absorption de solution est donc restée très superficielle. La pierre de Richemont, plus poreuse que celle de León, a montré, en revanche, une augmentation rapide de la masse qui a coüncidé avec une chute importante de la vitesse du son, laquelle, à la fin de l'essai, s'est maintenue à une valeur constante de 10 à 15\% de la valeur initiale. Ces évolutions indiquent clairement que la quantité de solution absorbée par la pierre de Richemont a rapidement imprégné la totalité des pores de l'échantillon.

Il apparait donc que les transferts de masse par évaporation et humidification s'établissent sur une zone 
la superficie y más estrecha que la correspondiente de Richemont.

La comparación de los valores de propagación determinados antes y al final del ensayo y tras el secado en estufa corrobora lo anterior: tras este secado, se produce la total cristalización de las sales en el espacio poroso, provocando una modificación en las propiedades acústicas de los materiales (Tabla 1). En el caso de León, las medidas de ultrasonidos antes del ensayo y tras el secado en estufa son similares lo que indica que la disolución penetra sólo hasta zonas cercanas a la superficie sin alcanzar el interior; la sal cristalizada tras el secado no afecta a la medida ultrasónica ya que no afecta al volumen global de la probeta. En Richemont, la velocidad de propagación después del secado en estufa es sensiblemente superior a la velocidad medida al inicio del ensayo. Esta variación indica que existe una cantidad importante de sal cristalizada en el interior de la probeta y por tanto que la disolución, durante el ensayo, alcanzó más profundidad.

\section{Análisis de las modificaciones superficiales por tratamiento de imágenes}

A continuación se presenta el estudio de la evolución del estado superficial de las probetas de ambos tipos de roca estudiada desde el punto de vista cualitativo, a partir de los mapas topográficos y de niveles de gris correspondientes al relieve (Figuras 4 y 5), y desde un punto de vista cuantitativo a través de la determinación y representación gráfica de la desviación típica extraída de las matrices numéricas del relieve y de la reflexión (Figura 6).

\section{Aspectos cualitativos del deterioro}

Los mapas topográficos (Figura 4) que representan cuatro etapas de la degradación de una probeta de la roca de León y de una de Richemont, permiten constatar la diferencia en intensidad y morfología de la desagregación arenosa que se desarrolla en ambas rocas.

Tras 9 días de ensayo se observa en la roca de León las primeras pérdidas localizadas de material superficial que alcanzan una profundidad de $1 \mathrm{~mm}$. La desagregación comienza a afectar enseguida al total de la superficie rocosa que muestra ya la presencia de pequeños alveolos. Richemont sufre, por el contrario, una desagregación más superficial, menos profunda, de varias décimas de milímetro entre cada etapa de análisis realizado. El total de la superficie se altera progresivamente bajo la forma de una fina desagregación arenosa. Al final del ensayo, la voisine de la surface plus réduite pour la pierre de Léon que pour le Richemont.

La comparaison des valeurs de vitesse du son mesurées après la phase de séchage démontre la même chose. Après séchage, la totalité des sels contenus à l'intérieur de l'espace poreux cristallise en entraînant une modification des propriétés acoustiques du matériau (Tableau 1). Dans le cas du León, les mesures effectuées avant l'essai et après le séchage donnent des résultats similaires, ce qui indique que le sel s'est accumulé à proximité de la surface et n'a pas atteint le coeur de l'échantillon. Pour la pierre de Richemont, en revanche, les vitesses du son mesurées après séchage sont sensiblement plus élevées que celles mesurées au début de l'essai. Cette variation témoigne de la présence en quantité importante de sel cristallisé au coeur de l'échantillon et donc, de la diffusion de la solution en profondeur au cours de l'essai de vieillissement.

\section{Analyse des altérations superficielles par le système laser-caméra}

L'évolution de l'état de surface des éprouvettes est comparée à l'aide du système de balayage laser décrit précédemment. Comme nous l'avons indiqué, la progression de l'altération peut être appréciée de manière qualitative à travers les représentations topographiques et en niveaux de gris des matrices de relief (Figures 4 et 5), et de manière quantitative, en utilisant le paramètre de dispersion statistique calculé à la fois sur les matrices de relief et de réflexion laser (Figure 6).

\section{Aspects qualitatifs}

Les graphiques topographiques de la Figure 4 représentent et comparent quatre étapes de la dégradation d'un échantillon de pierre León et d'un échantillon de Richemont. Ils mettent en évidence des différences sur l'intensité et morphologie de la désagrégation sableuse qui se développe sur la surface des deux pierres.

Après 9 jours de simulation, on observe sur la pierre de León quelques désagrégations profondes (>1 mm) mais localisées. Les désagrégations se généralisent ensuite à l'ensemble de la surface lui donnant un aspect alvéolaire déchiqueté, la perte de matière s'effectuant par amas plus ou moins importants. Le Richemont subit par contre une désagrégation plus superficielle, de quelques dixièmes de $\mathrm{mm}$ entre chaque étape d'analyse présentée. L'ensemble de la surface s'altère progressivement sous la forme d'une fine désagrégation sableuse. 

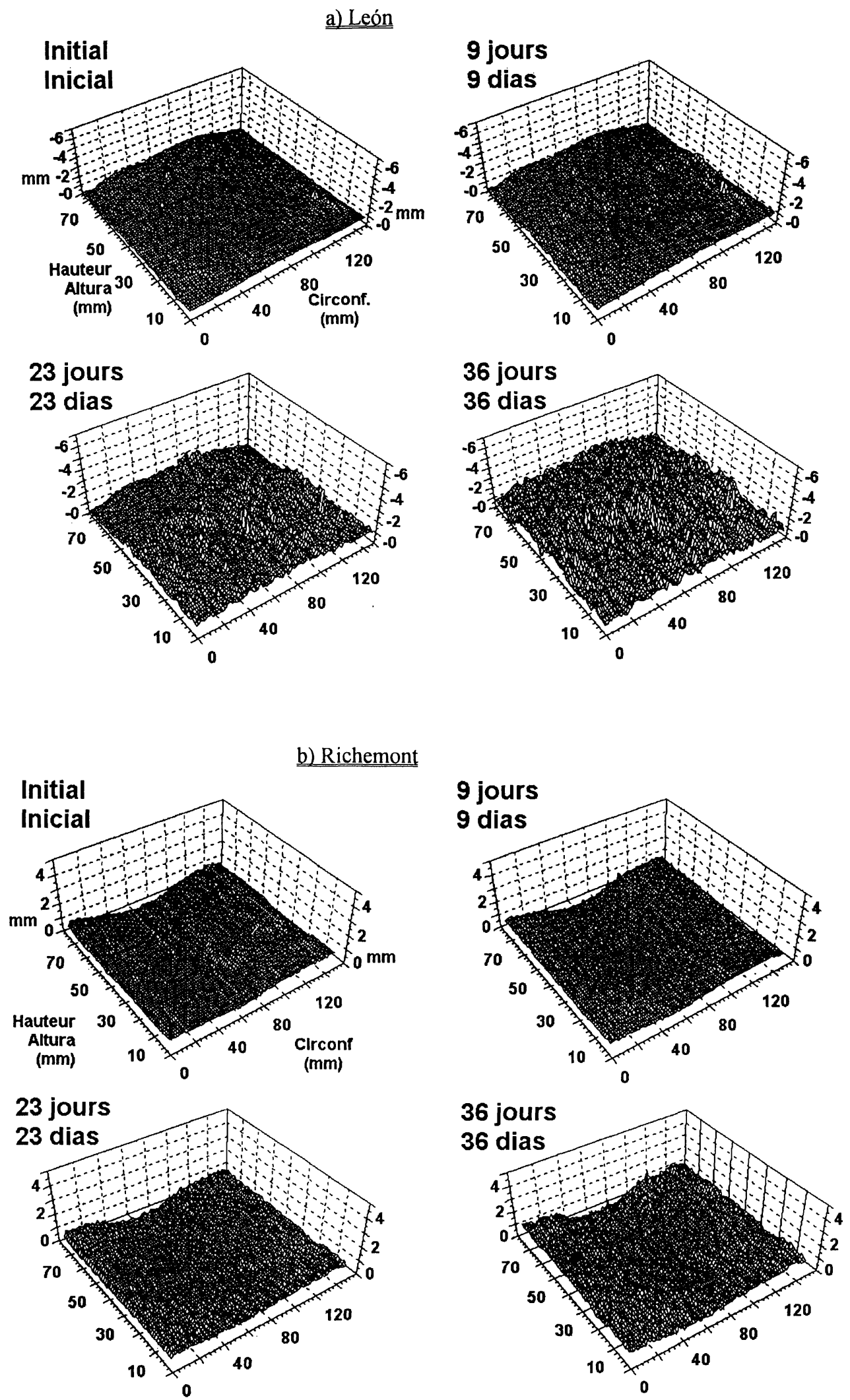

Figura 4.- Mapas topográficos de la superficie de una probeta de León (a) y de Richemont (b) en distintas etapas del ensayo de alteración artificial. Figure 4.- Graphiques topographiques de l'aspect de la surface d'un échantillon de León (a) et de Richemont (b) en cours d'altération. 

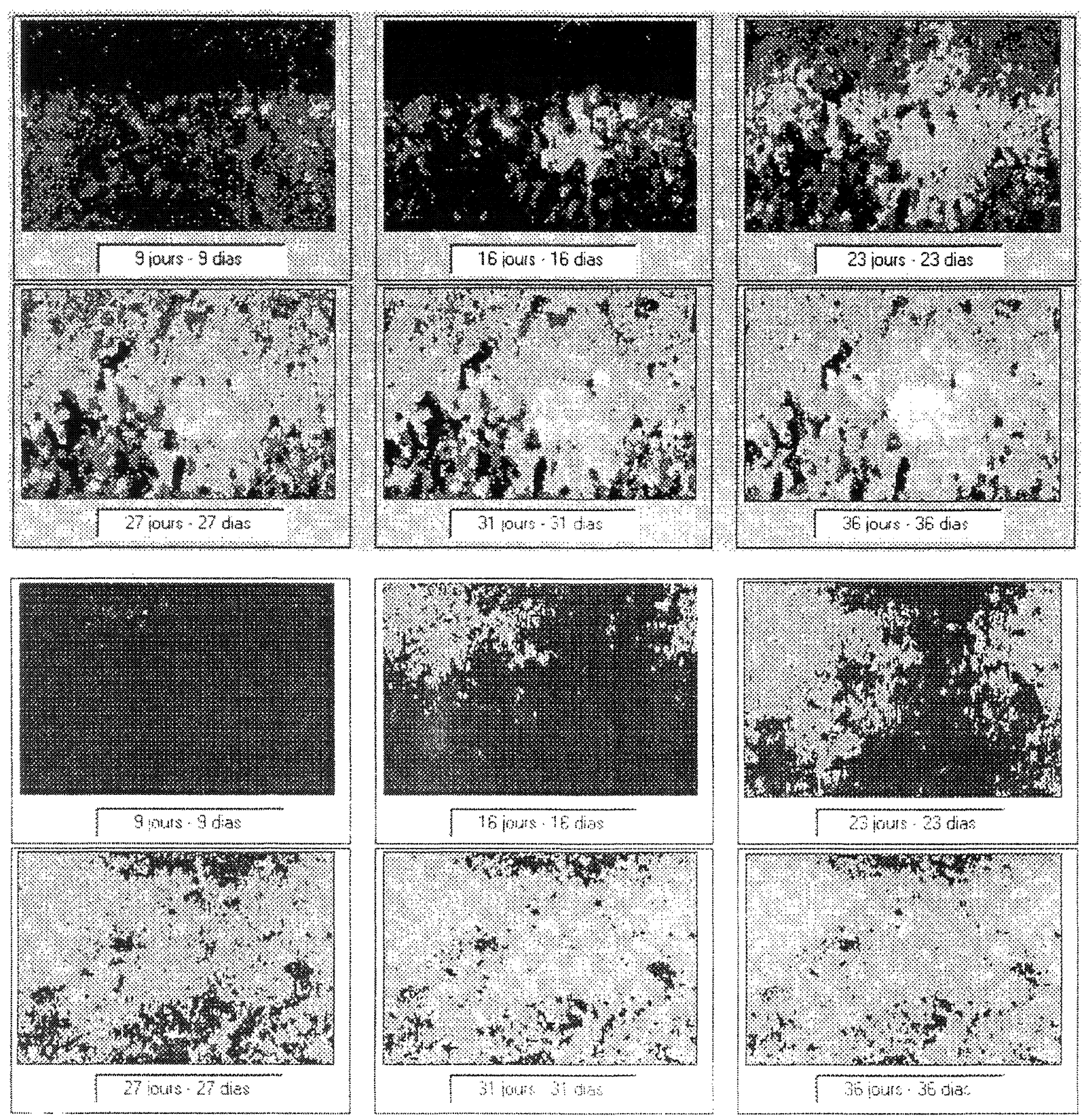

Figura 5.- Gráficos de niveles de gris que muestran la progresión de la desagregación arenosa de una probeta de León (a) y de Richemont (b)

Figure 5.- Graphiques en niveaux de gris montrant des désagrégations sableuses sur unéchantillon de León (a) et de Richemont (b).

rugosidad de la superficie de las probetas de León muestra amplitudes que alcanzan los $3 \mathrm{~mm}$ mientras que la de Richemont no sobrepasa $1 \mathrm{~mm}$.

Los gráficos de niveles de grises (Figura 5) permiten apreciar la evolución de la desagregación arenosa y su morfología a lo largo del ensayo, poniendo de manifiesto que ésta progresa de una manera irregular y heterogénea sobre la superficie de León mientras que lo hace de manera más homogénea en la piedra de Richemont.
Au terme de la simulation, les rugosités de surface de l'éprouvette de León atteignent parfois des amplitudes de l'ordre de $3 \mathrm{~mm}$ alors qu'elles ne dépassent pas $1 \mathrm{~mm}$ pour le Richemont

Avec les graphiques en niveaux de gris (Figure 5), il est facile d'apprécier l'étendue de la désagrégation et sa morphologie. Il apparaît clairement qu'elle progresse de manière irrégulière et par amas sur la surface des León alors qu'elle évolue de manière. plus homogène sur le Richemont. 

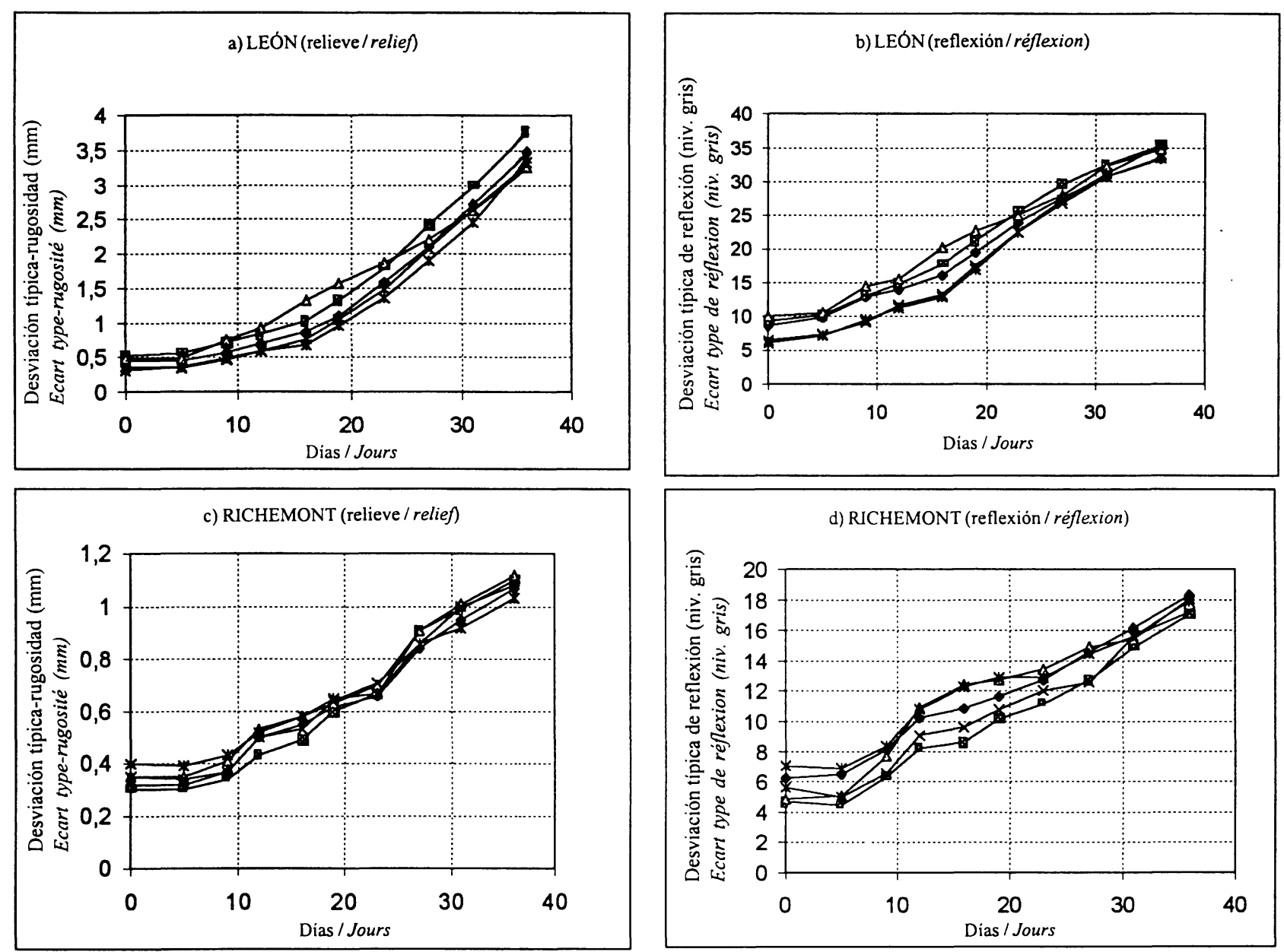

Figura 6.- Evolución a lo largo del ensayo de la desviación típica calculada a partir de las matrices de datos del análisis visual de las probetas de León (a relieve; b: reflexión) y de Richemont (c: relieve; d: reflexión).

Figure 6.- Courbes d'évolution du paramètre statistique de dispersion calculé sur les matrices de données visuelles pour les échantillons de León (a: relief; b: réflexion) et de Richemont (c: relief; d: réflexion).

La representación en niveles de grises permite también localizar rápidamente las zonas más dañadas, poniendo de manifiesto el carácter más superficial de la desagregación de Richemont frente al más profundo de la piedra de León.

\section{Aspectos cuantitativos}

Un tercer tratamiento de la información sobre la desagregación ofrecida por el análisis visual por láser consiste en la extracción de la desviación típica a partir de las matrices numéricas, la cual se representa en la Figura 6.

La evolución de la desviación típica de las matrices de relieve es bastante similar en ambos tipos de roca (Figura 6 a-c): en ambas rocas se aprecia un punto de inflexión, en torno a las dos semanas de ensayo, en el incremento de este parámetro, que es interpretado como un incremento en la rugosidad de las superficies como
La représentation en niveaux de gris permet aussi de localiser rapidement les zones les plus endommagées. Elle met notamment en évidence le caractère superficiel de la désagrégation du Richemont face à celle, par endroit beaucoup plus profonde, de la pierre de León.

\section{Aspects quantitatifs}

Une troisième approche permettant l'analyse de la désagrégation consiste donc à étudier l'évolution de l'écart type calculé sur les matrices numériques. Les résultats sont présentés sur les graphiques de la Figure 6.

Les étapes d'évolution du relief semblent au premier abord assez comparables pour les deux types de pierre (Figure 6 a-c): la désagrégation de la surface est perceptible dès la seconde semaine de l'essai ce qui se traduit sur le graphique par un accroissement du paramètre de rugosité. Puis, elle augmente 
consecuencia del desencadenamiento de la desagregación arenosa. En ambas rocas, este incremento se hace constante y se prolonga hasta el final del ensayo.

Sin embargo, los valores de este parámetro son diferentes para ambas rocas, lo que refleja la diferente intensidad de deterioro que sufren ambos materiales; los valores más elevados de la desviación típica en el caso de la roca de León reflejan una mayor rugosidad de la superficie. Estos valores son tres veces más elevados que en Richemont, relación comparable a la de la pérdida de peso constatada en la Figura 2 a-b.

En cuanto a la reflexión de la luz láser, la evolución de la desviación típica es diferente en cada roca (Figura 6 b-d). Con el fin de interpretar la evolución de este parámetro a lo largo del ensayo, es necesario tener en cuenta los diferentes fenómenos que pueden afectar la reflexión de la luz láser por las superficies rocosas:

- variaciones en el color de la superficie de las probetas relacionadas con la oxidación de las inclusiones metálicas o con la deposición de eflorescencias salinas.

- aparición de la desagregación arenosa que supone una modificación de la geometría superficial de la probeta y que, por tanto, afecta a la intensidad del haz reflejado.

- una profundidad de deterioro muy intensa puede dar lugar, incluso a la desaparición completa del haz láser reflejado.

Teniendo en cuenta estos fenómenos, los gráficos de la Figura 6 muestran lo siguiente:

- en el caso de la roca de León (Figura 6-b), este parámetro pone en evidencia la alteración más lenta durante las primeras dos semanas de ensayo y la posterior aceleración del deterioro a partir de decimoquinto día en que este parámetro sufre un incremento. Transcurrido un mes, este incremento se suaviza, lo que indica que la desagregación arenosa se ha generalizado por toda la superficie, si bien, la rugosidad sigue aumentando como lo muestra la evolución de la desviación típica del relieve.

- en el caso de Richemont (Figura 6-d), el brusco incremento de este parámetro tras cinco días de ensayo refleja la brusca variación de color de la superficie producida a partir del quinto día. El parámetro sufre una ligera modificación a partir de la segunda semana de simulación, momento en el cual aparecen las primeras manifestaciones de desagregación arenosa. progressivement, mais les niveaux d'altération sont cependant différents pour les deux types de pierre.

L'amplitude des désagrégations estimée à l'aide du critère de rugosité apparaît en effet trois fois plus élevée sur la pierre de León que sur la pierre de Richemont. Ce rapport est comparable au rapport des pertes de poids constatées sur les deux litho types (Figure $2 a-b$ ).

En ce qui concerne la réflexion de lumière laser, l'écart type calculé sur la matrice de réflexion met en évidence des différences assez importantes d'une pierre à l'autre (Figure 6 b-d). Afin d'interpréter l'évolution de l'écart type de la réflexion au cours de l'essai, il faut prendre en considération les différents phénomènes de surface susceptibles de modifier la réflexion de la lumière laser:

-les modifications de teintes liées aux dépôts cristallins ou à la corrosion d'éléments métalliques présents dans le matériau,

-l'apparition des premières désagrégations qui affectent l'intensité du faisceau réfléchi par une modification de la géométrie de la surface,

-lorsque la dégradation est très marquée, l'occultation du trait laser sur la surface qui tend à limiter l'écart type de la matrice de réflexion.

Compte tenu des ces observations, on peut identifier sur les graphiques de la Figure 6 les principales étapes qui marquent l'évolution de l'état de surface des pierres.

-pour les échantillons de León (Figure 6-b), les paramètres visuels mettent bien en évidence l'altération lente du matériau durant les deux premières semaines de l'essai puis l'accélération de la dégradation au-delà du

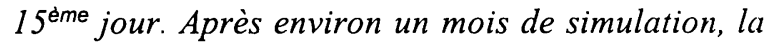
courbe de réflexion s'infléchit ce qui tend à montrer que les désagrégations se sont généralisées à l'ensemble de la surface, le relief continuant toujours à se creuser comme le montrent les courbes d'évolution du critère de rugosité du graphique 6-a.

-pour le Richemont (Figure 6-d), les courbes de réflexion mettent en évidence une brutale variation de teinte après 5 jours d'essai; elle résulte de la corrosion de particules métalliques réparties dans le matériau. Durant la deuxième semaine de simulation l'évolution du paramètre de dispersion de la réflexion s'est légèrement modifiée au moment où les premières manifestations de la désagrégation sont apparues. 
Concentración de $\mathrm{NaCl}$ (en $\mathrm{mg} / \mathrm{g}$ ) a diferentes profundidades desde la superficie de una probeta de Richemont y de León

(Concentration de $\mathrm{NaCl}$ (en $\mathrm{mg} / \mathrm{g}$ ) en fonction de la distance par rapport à la surface dans une éprouvette de Richemont et de León)

\begin{tabular}{|c|c|c|c|c|c|}
\hline $\begin{array}{c}\text { Profundidad } \\
\text { (Profondeur) }\end{array}$ & $0-3 \mathrm{~mm}$ & $4-6 \mathrm{~mm}$ & $7-10 \mathrm{~mm}$ & $>11 \mathrm{~mm}$ & $\begin{array}{c}\text { Roca sin alterar } \\
\text { (Pierre saine) }\end{array}$ \\
\hline Richemont & $21 \mathrm{mg} / \mathrm{g}$ & $18 \mathrm{mg} / \mathrm{g}$ & $20 \mathrm{mg} / \mathrm{g}$ & $6 \mathrm{mg} / \mathrm{g}$ & $0,7 \mathrm{mg} / \mathrm{g}$ \\
\hline León & $30 \mathrm{mg} / \mathrm{g}$ & $7 \mathrm{mg} / \mathrm{g}$ & $5 \mathrm{mg} / \mathrm{g}$ & $5 \mathrm{mg} / \mathrm{g}$ & $1 \mathrm{mg} / \mathrm{g}$ \\
\hline
\end{tabular}

\section{Análisis del contenido en $\mathrm{NaCl}$}

Los resultados de la extracción de ion cloruro que se presentan en la Tabla 2 confirman que la disolución salina alcanza mayor profundidad en Richemont. En León, los mayores contenidos de cloruro de sodio se encuentran en los cuatro primeros centímetros de profundidad, mientras que en Richemont la cantidad en $\mathrm{NaCl}$ se encuentra todavía en cantidades altas a mayores profundidades.

\section{DISCUSIÓN Y CONCLUSIONES}

La acción de las sales de procedencia marina ha provocado en ambas rocas la misma morfología de deterioro, es decir, desagregación arenosa, más o menos profunda, de la superficie. Esta forma de deterioro aparece asociada a sales de elevada solubilidad como el cloruro de sodio y es provocada por episodios alternantes de disolución-cristalización de la sal en el espacio poroso $(3,11,12,13)$.

Así, durante la pulverización de la niebla salina, la disolución se deposita sobre las superficies y penetra hacia el interior de la roca. Durante la fase de secado, la evaporación del agua desde la superficie incrementa la concentración de la disolución en el interior de la masa rocosa lo que favorece la cristalización de las sales. La presión que ejercen los cristales durante su formación sobre las paredes de los poros provoca la desestabilización del material y finalmente la desagregación de la roca.

Si bien el mecanismo de deterioro es el mismo en ambas rocas, la intensidad y la progresión de la desagregación a lo largo del ensayo difieren sensiblemente de una roca a otra. En este sentido, los métodos de análisis no destructivos aplicados en este trabajo han sido esenciales en el establecimiento de esta diferencia.

Así, tras un período de latencia, la piedra de León se desagrega rápidamente y de manera continuada sin que

\section{Analyse de la teneur en $\mathrm{NaCl}$}

Les résultats regroupés dans le Tableau 2 confirment bien la présence d'une quantité importante de $\mathrm{NaCl}$ à l'intérieur du Richemont tandis que la concentration en sel est restée faible dans la pierre de León, à l'exception de la zone superficielle.

\section{DISCUSSION ET CONCLUSIONS}

L'action des sels marins sur les deux pierres calcaires étudiées a provoqué la même morphologie d'altération: la désagrégation sableuse plus ou moins profonde de la surface. Il est acquis que cette détérioration a pour origine les processus alternatives de cristallisation et dissolution des sels, principalement le chlorure de sodium $(3,11,12,13)$.

Pendant la pulvérisation de brouillard salin, la solution saline se dépose sur la surface des pierres et pénètre vers l'intérieur de la roche puis, en phase de séchage, l'évaporation de l'eau en surface accroit la concentration saline de la solution ce qui favorise la cristallisation des sels. En exerçant des pressions sur les parois des pores, les croissances cristallines provoquent la désagrégation de la pierre.

Mais s'il est vrai que le mécanisme d'altération est commun pour les deux pierres, il faut néanmoins constater que l'intensité et la progression de la désagrégation au cours de l'essai diffèrent sensiblement pour chaque pierre. Les méthodes d'analyse appliquées dans ce travail ont été essentielles pour établir cette différence.

Après une période de latence, la pierre de León se désagrège rapidement et de manière continue, sans 
haya absorbido una gran cantidad de disolución y sin que ésta haya penetrado a gran profundidad en la roca. El análisis de sales solubles indica que se ha producido una acumulación de sales en los primeros milímetros de profundidad, indicando que los procesos de humectación y secado y la acción de las sales se establecen en esta roca en un área muy próxima a la superficie. La desagregación arenosa producida afecta a la globalidad de la superficie de una manera heterogénea debido a la heterogeneidad textural de la roca.

Richemont muestra un comportamiento diferente: tras los primeros ciclos del ensayo, las probetas tienden a saturarse progresivamente en la disolución de manera que ésta alcanza el interior de las probetas manifestándose en las medidas de velocidad de ultrasonidos. La estabilización posterior de los valores de propagación indican que se establece un equilibrio entre la humectación y el secado y es tras la aparición de la desagregación arenosa cuando en la evolución del peso comienza a observarse que la pérdida de material domina la ganancia por absorción de más disolución. El análisis visual muestra que la desagregación de esta roca es más lenta, menos profunda y más homogénea debido a su granulometría más fina.

Estos resultados muestran que la metodología no destructiva aplicada en este trabajo puede ser muy eficaz en estudios acerca del deterioro provocado durante ensayos de alteración artificial ya que permite una descripción objetiva de las transformaciones sufridas por los materiales y el establecimiento del comportamiento específico de cada roca. Esta metodología implica tres métodos de análisis complementarios, los cuales poseen, cada uno de ellos, una finalidad claramente definida:

. La medida de la variación de peso ofrece una información muy valiosa acerca de la cinética de absorción de la disolución salina al inicio del ensayo y es muy útil en la cuantificación del deterioro a través del porcentaje final de la pérdida de peso.

- La medida de la variación de la velocidad de propagación de ultrasonidos informa acerca de la capacidad de absorción de los materiales rocosos durante el ensayo, permite conocer si la disolución penetra hasta el interior de las rocas y, por tanto, junto con la medida de la variación de masa, poder establecer las diferencias que existen entre los materiales durante los procesos de humectación y secado. Por el contrario, esta técnica resulta insensible a las modificaciones que se producen en el sistema poroso como resultado de la acción de las sales durante el ensayo, ya que aquéllas se producen en zonas muy cercanas a la superficie. que la solution saline ait pénétré le matériau en profondeur et sans avoir absorbé une grande quantité de solution. L'analyse de la répartition des sels dans l'épaisseur des échantillons démontre qu'il en résulte une concentration saline importante localisée aux régions superficielles de la pierre. Les échanges de masse et l'action des sels s'établissent donc à proximité de la surface qui prend rapidement un aspect très déchiqueté compte tenu de l'hétérogénéité de la texture de la pierre León.

La pierre de Richemont montre un comportement plus complexe: après les premiers cycles de l'essai les éprouvettes tendent progressivement à se saturer par diffusion de solution dans l'ensemble de leur volume ce qui modifie sensiblement les propriétés acoustiques du matériau. La stabilisation de la vitesse du son démontre qu'il s'établit ensuite un équilibre dans les échanges par humidification et évaporation avec le milieu extérieur. Après l'amorçage du processus de désagrégation, le bilan de masse est dominé par la perte de matière. La dégradation est plus lente, plus homogène compte tenu de la granulométrie plus fine du Richemont, et moins profonde que celle constatée sur la pierre de León.

Les moyens d'analyse mis en ouvre ont permis de suivre efficacement les transformations subies par les matériaux et de cerner les spécificités du processus d'altération pour ces deux pierres. Le travail présenté dans cet article permet donc de dégager une méthodologie expérimentale pour l'étude du processus d'altération artificielle par brouillard salin des pierres de construction. Cette méthodologie implique trois moyens d'analyse non destructifs complémentaires qui ont chacun une finalité clairement définie.

La variation de masse fournit de précieuse indication sur la cinétique d'absorption de la solution au début de l'essai de vieillissement. Le pourcentage final de perte de masse fournit aussi une donnée quantitative sur le niveau de désagrégation subi.

\section{- La mesure acoustique permet de déterminer à quel} moment la solution a atteint le matériau à coeur. Elle constitue en ce sens une information complémentaire à la mesure de variation de masse pour comparer le comportement des pierres, dans leur volume, sous l'effet des phases l'humidification et de séchage. La mesure acoustique reste en revanche insensible à la désagrégation du matériau puisque ce phénomène d'altération est le résultat de processus de cristallisation et dissolution très superficiels qui n'affectent pas la profondeur de la pierre. 
Las modificaciones que ocurren en la superficie de los materiales son, sin embargo, analizadas gracias al sistema de barrido con luz láser, que además de una descripción objetiva de la morfología permite cuantificar la intensidad y la profundidad que alcanza. El cálculo de la desviación típica a partir de las matrices numéricas constituye, por otra parte, una herramienta muy útil para establecer la cinética de desagregación; la desviación típica calculada a partir de las matrices de relieve, que evoluciona, además, de manera proporcional a la variación de peso, resultó muy adecuada como parámetro de rugosidad de la superficie alterada. La desviación típica a partir de las matrices de reflexión, además de aportar una información complementaria a la del análisis del relieve, permite detectar variaciones de color de la superficie como consecuencia de la alteración

\section{AGRADECIMIENTOS}

Este trabajo fue desarrollado dentro del Proyecto E.C. Project, Contract $n^{\circ}$ ENV4-CT95-0100.
L'analyse de l'évolution de l'état de surface par un système de balayage laser-caméra permet de décrire objectivement la morphologie d'altération, son intensité et profondeur. De plus, le calcul de l'écart type sur les matrices de données visuelles fournit un critère d'évaluation global adapté pour comparer les cinétiques de désagrégation des surfaces. Dans le cas de l'analyse du relief, ce paramètre statistique évolue dans les mêmes proportions que la perte de masse et il est équivalent à un critère de rugosité de surface. La matrice de réflexion laser apporte des éléments d'informations complémentaires à l'analyse du relief, l'écart type calculé sur cette matrice fournit un critère d'altération qui évolue de la même façon que le critère de rugosité lorsque la surface se désagrège. La réflexion laser peut aussi être exploitée pour analyser des formes de détérioration qui provoquent des variations de teinte de la surface (auréoles, dépôts, corrosion).

\section{REMERCIEMENTS}

Ce travail a été réalisé grâce au soutien financier du programme européen EC Project ENV4-CT95-0100.

\section{BIBLIOGRAFÍA}

(1) Auger, F., Simulation et étude des phénomènes de dégradation des roches calcaires en milieu aérien marin', Proceeding of RILEM Conference on the Durability of Construction Materials, organized by AFREM, Versailles, France, sept. 1987,911-914.

(2) Auger, F., Méthodologie d'utilisation de la vitesse du son en vue de l'étude de l'altération des roches calcaires, Proceeding $3^{\text {cme journcee }}$ Universitaire de Géotechnique, St-Nazaire (France) 28-30 janvier(1987), 323-330.

(3) Auger, F., Simulation accélérée de la dégradation des matériaux de construction en ambiance aérienne saline, Proceeding of IAEG (Greek) Conference on the Engineering Geology of Ancient Works, Monument and Historical Sites, Athens, (1988), 797-804.

(4) Auger, F., Influence des fluides interstitiels sur la vitesse du son dans les matériaux de construction. Mesures expérimentales et conséquences sur les diagnostics d'altérabilité. Proceeding Conf. Int : Mesure et Essais en Génie Civil, Lyon-Villeurbanne 13-16 sept.,vol. 2,1988 , p. 797-804.

(5) Auger F., Vieillissement par altération atmosphérique des matériaux de construction-Etude comparative in situ et en simulation. Proc of Int. Symp. on the Deterioration of Building Materials, La Rochelle, 12-14 june 1991, 115-128.

(6) Birginie, J.-M.; Rédarce, T and Jutard, A., Using a camera-laser association to detect skin edges in a tannery, RAPA, Revue d'Automatique et de Productique Appliquée, 6(1993), 43-63.

(7) Birginie, J-M; Auger, F., Caractérisation visuelle de l'altération d'échantillons carottés de pierres, vicillis en simulation dynamique aux brouillards salins, Materials and Structures, Vol. 32, 1999, 584-592.

(8) Boubie, T.; Coussy, O.; Zinszner, B., Acoustique des milieux poreux. Ed. Technip, publication de L'Institut français du Pétrole, Coll. Science et technique du pétrole, 27,339p., 1986.

(9) Bournazel, J-P.; Hermida, G.; Martinet, G., Etude de la dégradation des maçonneries des greniers à scl de Honfleur par action du chlorure de sodium, Revue Française de Génie Civil, Vol. 1; 1 (1997), 115-136.

(10) Esbert, R.; Ordaz, J.; Alonso, F.J.; Alba, J.M., (1981). Petrographic and physical study of the building stones from León Cathedral (Spain). The Conservation of Stone, II. Preprints of the Constributions to the International Symposium, Bologna, 27-30 October 1981. RossiManaressi (Ed.) Part A, Deterioration. 285-298.

(11) Iniguez Herrero J., (1967). Altération des calcaires et des grès utilisés dans la construction. Thèse (1961) traduite par J. Stichelbaudt. Éd. Eyrolles, 61 Boulevard St Germain, Paris.

(12) Rivas, T.,(1997) Mecanismos de alteración de rocas graníticas utilizadas en la construcción de edificios antiguos en Galicia. Tesis Doctoral. Servicio de Publicacións e Intercambio Científico. Universidade de Santiago, 365 pp.

(13) Silva, B. ; Rivas, T. ; Prieto, B., (1996).- Relation between type of soluble salt and decay forms in granitic coastal churches in Galicia (NW Spain). In European Commision Research Workshop Origin, Mechanisms and Effects of Salts on Degradation of Monuments in Marine and Continental Environments. DGII Protection and Conservation of the European Cultural heritage, research Report 4, pp 181-190, 1996. 